\title{
AVALIAÇÃO DE PACIENTES COM DISTROFIA MUSCULAR DE DUCHENNE EM LABORATÓRIO COMPUTADORIZADO DE MARCHA ATRAVÉS DO ÍNDICE DE ALTERAÇÃO DA MARCHA
}

\author{
EVALUATION OF PATIENTS WITH DUCHENNE MUSCULAR DYSTROPHY \\ IN GAIT ANALYSIS LABORATORY USING GAIT DEVIATION INDEX
}

\begin{abstract}
Alessandro Giurizatto Melanda' ${ }^{1}$, Ana Carolina Pauleto', Luiz Alberto Knaut ${ }^{2}$, Alana Elisabeth Kuntze Ferreira ${ }^{3}$,
\end{abstract} Dielise Debona lucksch², Rauce Marçal da Silva ${ }^{3}$, Rodrigo Faucz Munhoz da Cunha ${ }^{4}$

\section{RESUMO}

Objetivo: Aplicação e correlação do índice de alteração da marcha (GDI) com questionário de avaliação funcional (FAQ), escala de mobilidade funcional (FMS) e índice de Sutherland (IS) para pacientes com distrofia muscular de Duchenne (DMD). Métodos: Onze crianças com diagnóstico clínico de DMD, idade entre 6-11 anos, deambuladoras, participaram do estudo. O GDI foi correlacionado com o FAQ, FMS e IS, utilizando o coeficiente de correlação rho de Spearman-Rank. O GDI foi calculado com a cinemática dos membros inferiores coletada com seis câmeras Hawks da Motion Analysis Corporation. Resultados: O GDI médio obtido foi de $82,5( \pm 13,5)$, sendo este valor menor do que o esperado para indivíduos sem patologias musculoesqueléticas (i.e. $\geq 100$ ). O GDI apresentou correlação moderada com o FAQ e FMS $(50 / 500 \mathrm{~m})$ e forte com IS ( $r h o>0,83, \mathrm{p} \leq 0,05)$. Conclusão: O GDI apresenta correlação com classificações, sejam elas clínicas (i.e. FMS), por estudo do movimento (i.e. IS) e com questionário de avaliação funcional (i.e. FAQ).

Descritores - Distrofia Muscular de Duchenne; Marcha; Questionário

\section{ABSTRACT}

Objective: Aplication and correlation at Gait Deviation Index (GDI) and Functional Assessment Questionnaire (FAQ), Functional Mobility Scale (FMS) and Sutherland Index (SI) for patients with DMD. Methods: Eleven children with clinical diagnosis at Duchenne Muscular Dystrophy (DMD), between 6 and 11 years old, walkers, participated at study. The GDI was correlated with the FAQ, FMS and IS, using the rho coefficient of correlation of Spearman-Rank. To calculate the GDI, the kinematics were collected by six Hawks cameras (Motion Analysis Corporation). Results: The average GDI achieved was 82.5 (plus or minus 13.5), this value being lower than expected for individuals without musculoskeletal disorders (ie greater than or equal to 100). GDI showed moderate correlation with the FMS and FAQ (50/500m) and strong with IS (rho $>0.83, p<=0.05)$. Conclusion: The GDI shows correlation with clinical classifications (FMS), gait analysis classifications (IS) and questionnaries of function evaluation (FAQ).

Keywords - Muscular Dystrophy, Duchenne; Gait; Questionnaires

É uma desordem recessiva ligada ao cromossomo $\mathrm{X}$ devido a mutações no gene responsável pela produção da proteína distrofina ${ }^{(2)}$.

Alterações no sistema locomotor como fraqueza muscular progressiva, fadiga muscular e desenvolvimento

\footnotetext{
1 - Mestre em Medicina e Ortopedista Pediátrico do Laboratório de Marcha do Centro Hospitalar de Reabilitação Ana Carolina de Moura Xavier - Curitiba, PR, Brasil.

2 - Mestre e Fisioterapeuta do Laboratório de Marcha do Centro Hospitalar de Reabilitação Ana Carolina de Moura Xavier - Curitiba, PR, Brasil.

3 - Fisioterapeuta do Laboratório de Marcha do Centro Hospitalar de Reabilitação Ana Carolina de Moura Xavier - Curitiba, PR, Brasil.

4 - Engenheiro do Laboratório de Marcha do Centro Hospitalar de Reabilitação Ana Carolina de Moura Xavier - Curitiba, PR, Brasil.

Trabalho realizado no Centro Hospitalar de Reabilitação Ana Carolina de Moura Xavier - Curitiba, PR.

Correspondência: Rua Senador Souza Naves, 1.591 - 86010-160 - Londrina, PR. E-mail: melanda@uel.br

Trabalho recebido para publicação: 24/08/2011, aceito para publicação: 31/08/2011.
}

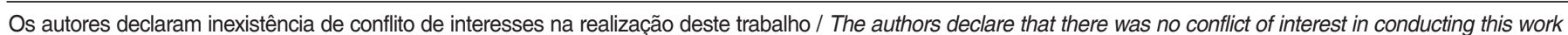


de contraturas articulares, alteram a marcha de crianças com DMD até perda desta habilidade entre a primeira e a segunda décadas de vida ${ }^{(3-5)}$. Com a evolução da terapia gênica, novas modalidades terapêuticas surgem para auxiliar o manuseio desta patologia ${ }^{(6)}$. A mensuração dos resultados funcionais destes tratamentos pode ser realizada com a avaliação da marcha. A análise computadorizada da marcha é um instrumento efetivo para avaliar e quantificar mudanças no andar de pacientes ${ }^{(7)}$. Entretanto, mesmo com a assistência da análise da marcha e a quantificação objetiva de mudanças, sejam elas decorrentes de intervenções ou da própria evolução do paciente, podem ser desafiadoras ${ }^{(8)}$. A utilização do índice de alteração da marcha (gait deviation index) possibilita quantificar a qualidade global da marcha através de uma escala numérica ${ }^{(9)}$.

O presente estudo tem como objetivo avaliar a aplicação do índice de alteração da marcha (GDI) em pacientes com DMD e sua correlação com instrumentos de avaliação funcional.

\section{MATERIAL E MÉTODOS}

Avaliados pacientes deambuladores com diagnóstico clínico de distrofia muscular de Duchenne, no Laboratório de Marcha do Centro Hospitalar de Reabilitação em Curitiba. Os pacientes foram orientados a caminhar com velocidade selecionada livremente em pista de 10 metros. Marcadores reflexivos foram colados na pele (Figura 1), de acordo com o modelo biomecânico Hellen-Hayes ${ }^{(7)}$.

Coletado movimento dos membros inferiores durante a marcha com uso de seis câmeras Hawks da Motion Analysis Corporation, Santa Rosa, CA. O GDI foi calculado utilizando os ângulos de movimento da pelve e do quadril nos planos sagital, coronal e transverso, flexão plantar e dorsiflexão dos tornozelos e o ângulo de progressão dos pés (Figura 2). Os dados foram extraídos a cada $2 \%$ do ciclo da marcha, com nove ângulos e 51 pontos, totalizando 459 dados. O GDI maior ou igual a 100 indica que a marcha do indivíduo é, no mínimo, próxima da média dos sujeitos tipicamente desenvolvidos ou sem patologias da marcha. Cada 10 pontos abaixo do valor 100 correspondem a um desvio padrão dos sujeitos tipicamente desenvolvidos.

O GDI foi correlacionado com faixa etária, índice de Sutherland ${ }^{(10)}$, questionário de avaliação funcional (functional assessment questionnaire $)^{(11)}$ e escala de mobilidade funcional (functional mobility scale) ${ }^{(12)}$, utilizando o coeficiente de correlação rho $(\rho)$ de Spearman-Rank.

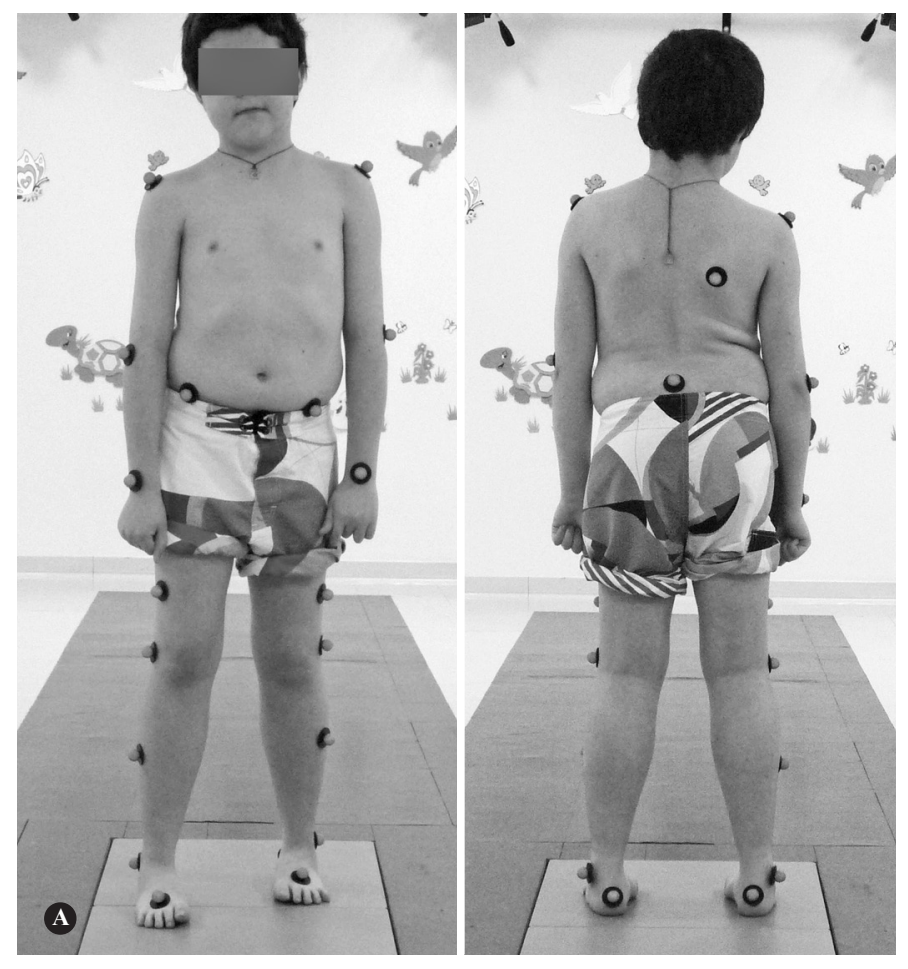

Figura 1 - Paciente de 10 anos com DMD. Nota-se hipertrofia das panturrilhas. Marcadores reflexivos foram colados na pele, de acordo com o modelo biomecânico Hellen-Hayes, para coleta de dados em laboratório computadorizado de marcha (A e B).
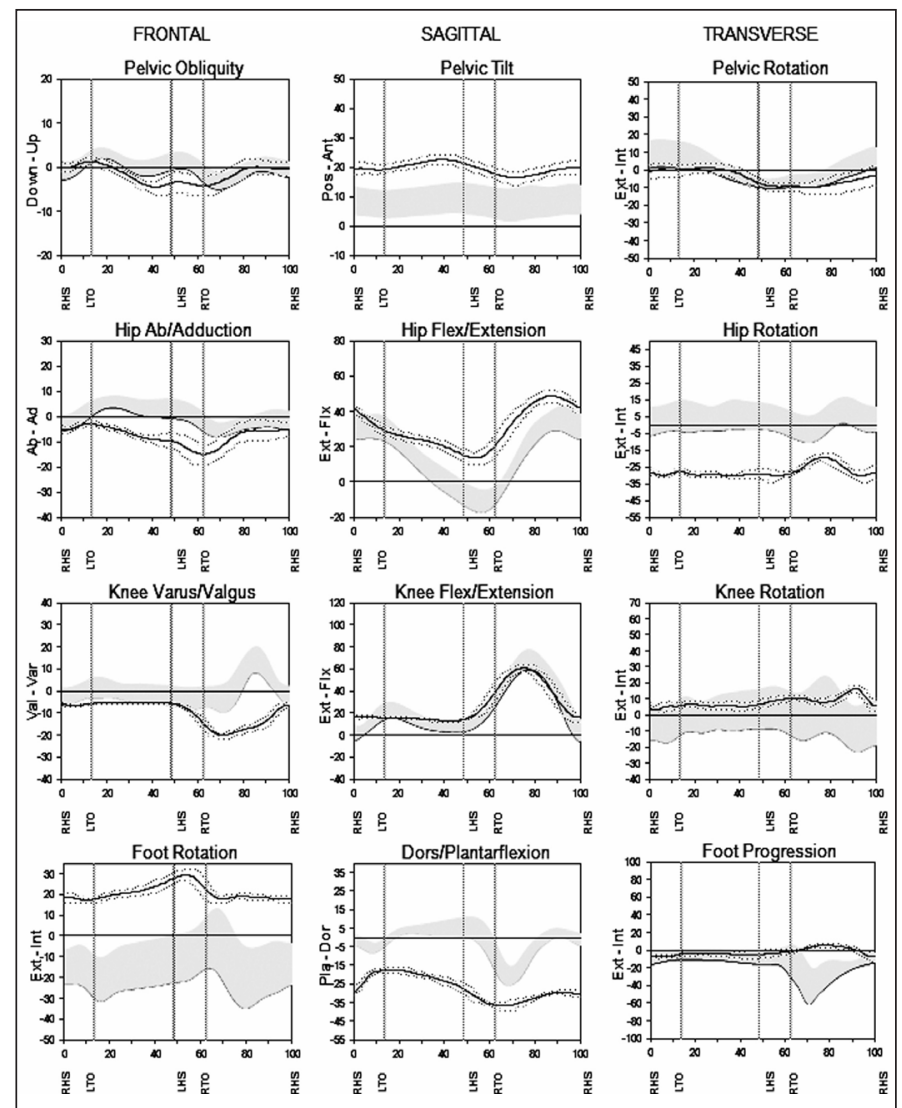

Figura 2 - Gráficos de cinemática dos membros inferiores nos planos coronal, sagital e transverso (colunas verticais). As colunas horizontais correspondem aos movimentos da pelve, quadril, joelho e tornozelo/pé durante o ciclo da marcha. 


\section{RESULTADOS}

Avaliados 11 indivíduos do sexo masculino entre seis e 11 anos de idade com diagnóstico de DMD.

O GDI médio foi de $82,5( \pm 13,5)$, sendo valores $\geq$ 100 esperados para normais. O GDI apresentou correlação moderada com faixa etária, FAQ, FMS (50/500m) e forte com IS $(\rho>0,83$ e $\mathrm{p} \leq 0,05)$ (Tabela 1$)$.

Tabela 1 - Correlação do índice de alteração da marcha (GDI) com as escalas índice de Sutherland (IS), questionário de avaliação funcional (FAQ), escala de mobilidade funcional (FMS) e faixa etária.

Coeficiente de correlação rho de Spearman-Rank - $p$ (significância) $n=11$

\begin{tabular}{c|c|c|c|c|c|c|c}
\hline & IS (D) & IS (E) & FAQ & FMS (5) & $\begin{array}{c}\text { FMS } \\
\mathbf{( 5 0 )}\end{array}$ & $\begin{array}{c}\text { FMS } \\
\mathbf{( 5 0 0 )}\end{array}$ & Idade \\
\hline GDI (D) & $\begin{array}{c}0,845 \\
(0,001)\end{array}$ & $\begin{array}{c}0,755 \\
(0,007)\end{array}$ & $\begin{array}{c}0,665 \\
(0,026)\end{array}$ & $\begin{array}{c}-0,060 \\
(0,861)\end{array}$ & $\begin{array}{c}0,551 \\
(0,079)\end{array}$ & $\begin{array}{c}0,337 \\
(0,311)\end{array}$ & $\begin{array}{c}-0,526 \\
(0,097)\end{array}$ \\
\hline GDI (E) & $\begin{array}{c}0,882 \\
(0,000)\end{array}$ & $\begin{array}{c}0,827 \\
(0,002)\end{array}$ & $\begin{array}{c}0,430 \\
(0,186)\end{array}$ & $\begin{array}{c}0,299 \\
(0,372)\end{array}$ & $\begin{array}{c}0,783 \\
(0,004)\end{array}$ & $\begin{array}{c}0,607 \\
(0,048)\end{array}$ & $\begin{array}{c}-0,340 \\
(0,307)\end{array}$ \\
\hline \\
\hline
\end{tabular}

\section{DISCUSSÃO}

A primeira descrição na literatura médica sobre a DMD foi feita em 1851 por Meryon, médico inglês, que reconheceu a natureza familiar, predileção pelo sexo masculino e o curso progressivo e finalmente fatal da doença ${ }^{(13)}$. Duchenne, o autor que leva o nome da afecção, publicou em 1858 descrição mais completa de 13 casos com informações histológicas obtidas de biópsias muscu$\operatorname{lares}^{(14)}$. Desde então, a DMD tem sido estudada sem que um tratamento efetivo altere o curso da doença.

A utilização de corticosteroides parece reduzir a expectativa da perda de força muscular com o passar do tempo ${ }^{(15)}$, mas não alteram a história natural do quadro. A terapia genética pode ser uma modalidade de tratamento mais eficiente, mas ainda encontra-se em pesquisa ${ }^{(16)}$.

Paralelamente às tentativas de desenvolver tratamentos que possam efetivamente produzir mudanças na história natural do quadro, estão as avaliações funcionais. A mensuração do impacto de uma intervenção é fundamental para acessar a efetividade deste tratamento. Para acessar a evolução motora dos pacientes com DMD utilizam-se escalas com ênfase na avaliação do grau de força muscular ${ }^{(17)}$. Entretanto, a subjetividade destes exames pode não refletir com fidedignidade a evolução motora do paciente.

O GDI é uma ferramenta desenvolvida, utilizando dados da análise computadorizada da marcha, para quantificar de maneira global a capacidade de deambulação do indivíduo ${ }^{(9)}$. Diversos trabalhos têm utilizado o GDI como ferramenta para avaliar os resultados das intervenções propostas, sejam elas medicamentosas ou cirúrgicas $^{(18,19)}$. Truong et al ${ }^{(20)}$ utilizaram o GDI para avaliar o resultado da cirurgia de alongamento do psoas em pacientes com paralisia cerebral.

A importância de índices que possibilitam a mensuração global da deambulação está no fato do impacto da mudança, após uma intervenção, poder não ser detectada diretamente no local da intervenção. Ao utilizar dados de vários segmentos (pelve, quadril, joelho, tornozelo e pé), o GDI tem capacidade de identificar modificações em vários níveis dos membros inferiores. Um exemplo desta situação foi observado no trabalho de Sienko et $a l^{(19)}$, que, durante a avaliação de pacientes com DMD, notaram que as mudanças decorrentes do uso de corticosteroides foram detectadas pelo GDI e não pelo padrão de deambulação e medidas das habilidades motoras.

A utilização de questionários respondidos por parentes ou cuidadores de crianças com alterações neuromusculoesqueléticas, como o questionário de avaliação funcional (FAQ), tem proporcionado ao profissional assistente entender como o paciente é no seu cotidiano. Enquanto a escala de mobilidade funcional (FMS) é utilizada para classificar a capacidade de locomoção, para distâncias de cinco, 50 e 500 metros, e facilitar a comunicação entre profissionais, além de apontar mudanças no decorrer do tempo.

Em 1981, Sutherland et al ${ }^{(10)}$ publicaram estudo de 21 pacientes com DMD, avaliados em laboratório de marcha com equipamento disponível na época (Vanguard Motion Analyzer). Os autores determinaram um índice evolutivo para a marcha dos pacientes com DMD. O índice é calculado através de três variáveis: máxima inclinação da pelve durante o ciclo da marcha, máxima dorsiflexão do tornozelo no balanço e cadência da marcha (número de passos por minuto). Com essas variáveis, o autor classificava o estágio evolutivo da marcha destes sujeitos em: inicial, intermediário e tardio. O índice de alteração da marcha (GDI) é calculado através de nove variáveis, as quais são coletadas com equipamento tridimensional (Motion Analysis Corporation, Santa Rosa, CA). Dessa forma, uma quantidade maior de dados são coletados e com equipamento mais adequado, o que parece fornecer uma caracterização mais efetiva do andar dos pacientes.

O GDI foi avaliado por Schwartz e Rozumalski ${ }^{(9)}$, em 
relação ao questionário de avaliação funcional (FAQ), escala de mobilidade funcional (FMS) em pacientes com paralisia cerebral $^{(9)}$ e mostrou-se um instrumento efetivo para mensuração da marcha daquela população. A utilização do GDI em pacientes com DMD foi relatada por Sienko et $a l^{(19)}$, que mostraram utilidade no método para diferenciar estágios da evolução da doença. No presente estudo, o GDI mostrou correlação com classificações, sejam elas clínicas (FMS), por estudo do movimento (IS) e com questionário de avaliação funcional (FAQ).

O estudo apresenta como principais limitações o número de pacientes e o fato de terem sido submetidos a apenas uma avaliação. O acompanhamento destes pacientes com novas avaliações associado a um maior número de indivíduos avaliados pode, no futuro, ampliar a utilidade da ferramenta (GDI).

\section{REFERÊNCIAS}

1. D’Angelo MG, Berti M, Piccinini L, Romei M, Guglieri M, Bonato S, et al. Gait pattern in Duchenne muscular dystrophy. Gait Posture. 2009;29(1):36-41.

2. Armand S, Mercier M, Watelain E, Patte K, Pelissier J, Rivier F. A comparison of gait in spinal muscular atrophy, type II and Duchenne muscular dystrophy. Gait Posture. 2005;21(4):369-78.

3. McDonald CM, Abresch RT, Carter GT, Fowler WM Jr, Johnson ER, Kilmer DD, et al. Profiles of neuromuscular diseases. Duchenne muscular dystrophy. Am J Phys Med Rehabil. 1995;74(5 Suppl):S70-92.

4. Roland EK. Muscular dystrophy. Pediatr Rev. 2000;21(7):233-7.

5. Gaudreault N, Gravel D, Nadeau S, Houde S, Gagnon D. Gait patterns comparison of children with Duchenne muscular dystrophy to those of control subjects considering the effect of gait velocity. Gait Posture. 2010;32(3):342-7.

6. Pichavant C, Aartsma-Rus A, Clemens PR, Davies KE, Dickson G, Takeda S, et al. Current status of pharmaceutical and genetic therapeutic approaches to treat DMD. Mol Ther. 2011;19(5):830-40.

7. Davis RB 3rd, Ounpuu S, Tyburski D, Gage JR. A gait analysis data collection and reduction technique. Human Movement Sci. 1991;10:575-87.

8. Schutte LM, Narayanan U, Stout JL, Selber P, Gage JR, Schwartz MH. An index for quantifying deviations from normal gait. Gait Posture. 2000;11(1):25-31.

9. Schwartz $\mathrm{MH}$, Rozumalski A. The gait deviation index: a new comprehensive index of gait pathology. Gait Posture. 2008;28(3):351-7.

10. Sutherland $\mathrm{DH}$, Olshen R, Cooper L, Wyatt M, Leach J, Mubarak S, et al The pathomechanics of gait in Duchenne muscular dystrophy. Dev Med Child Neurol. 1981;23(1):3-22.

11. Novacheck TF, Stout JL, Tervo R. Reliability and validity of the Gillette Func-

\section{CONCLUSÃO}

A distrofia muscular de Duchenne continua desafiando a medicina para a produção de um tratamento eficiente. $\mathrm{O}$ índice de avaliação da marcha (GDI) mostra-se ferramenta com capacidade de mensurar a marcha objetivamente e produzir um índice numérico único, como uma nota para a deambulação do indivíduo. Desta forma, a análise computadorizada da marcha pode contribuir na avaliação dos novos tratamentos para a DMD.

\section{AGRADECIMENTOS}

Os autores agradecem ao engenheiro Wagner de Godoy e Dr. Paulo Lucareli do Laboratório de Estudo do Movimento do Hospital Albert Einstein de São Paulo, pela constante colaboração e compartilhamento de conhecimentos.

tional Assessment Questionnaire as an outcome measure in children with walking disabilities. J Pediatr Orthop. 2000;20(1):75-81.

12. Graham HK, Harvey A, Rodda J, Nattrass GR, Pirpiris M. The Functional Mobility Scale (FMS). J Pediatr Orthop. 2004;24(5):514-20.

13. Hoffman EP, Fischbeck KH, Brown RH, Johnson M, Medori R, Loike JD, et al. Characterization of dystrophin in muscle-biopsy specimens from patients with Duchenne's or Becker's muscular dystrophy. N Engl J Med. 1988 May 26;318(21):1363-8.

14. Jay V. On a historical note: Duchenne of Boulogne. Pediatr Dev Pathol. 1998;1(3):254-5.

15. Sussman M. Duchenne muscular dystrophy. J Am Acad Orthop Surg. 2002;10(2):138-51.

16. Goyenvalle A, Seto JT, Davies KE, Chamberlain J. Therapeutic approaches to muscular dystrophy. Hum Mol Genet. 2011;20(R1):R69-78.

17. Brooke MH, Griggs RC, Mendell JR, Fenichel GM, Shumate JB, Pellegrino RJ. Clinical trial in Duchenne dystrophy. I. The design of the protocol. Muscle Nerve. 1981;4(3):186-97.

18. Cimolin V, Galli M, Vimercati SL, Albertini G. Use of the Gait Deviation Index for the assessment of gastrocnemius fascia lengthening in children with Cerebral Palsy. Res Dev Disabil. 2011;32(1):377-81.

19. Sienko Thomas S, Buckon CE, Nicorici A, Bagley A, McDonald CM, Sussman MD. Classification of the gait patterns of boys with Duchenne muscular dystrophy and their relationship to function. J Child Neurol. 2010;25(9):1103-9.

20. Truong WH, Rozumalski A, Novacheck TF, Beattie C, Schwartz MH. Evaluation of conventional selection criteria for psoas lengthening for individuals with cerebral palsy: a retrospective, case-controlled study. J Pediatr Orthop. 2011;31(5):534-40. 